\title{
Spatial and temporal filtering of a 10-W Nd:YAG laser with a Fabry-Perot ring-cavity premode cleaner
}

\author{
B. Willke, N. Uehara, E. K. Gustafson, and R. L. Byer \\ Edward L. Ginzton Laboratory, Stanford University, Stanford, California 94305-4085 \\ P. J. King, S. U. Seel, and R. L. Savage, Jr. \\ LIGO Project, MS 18-43, California Institute of Technology, Pasadena, California 91125
}

Received June 29, 1998

\begin{abstract}
We report on the use of a fixed-spacer Fabry-Perot ring cavity to filter spatially and temporally a 10 -W laser-diode-pumped Nd:YAG master-oscillator power amplifier. The spatial filtering leads to a 7.6-W TEM 00 beam with $0.1 \%$ higher-order transverse mode content. The temporal filtering reduces the relative power fluctuations at $10 \mathrm{MHz}$ to $2.8 \times 10^{-9} / \sqrt{\mathrm{Hz}}$, which is $1 \mathrm{~dB}$ above the shot-noise limit for $50 \mathrm{~mA}$ of detected photocurrent. (c) 1998 Optical Society of America

OCIS codes: $120.2230,330.6610,330.6790,140.3530$
\end{abstract}

In recent years the need for high-power lasers with good spatial and temporal characteristics has increased, with applications in laser machining, medicine, quantum optics, and gravitational-wave interferometry. Good pointing stability as well as pure Gaussian profiles are the key features for precision machining, whereas pure spatial profiles and power fluctuations near the shot-noise limit are crucial in gravitational-wave interferometry. Spatially and temporally filtered laser-diode-pumped solid-state lasers are promising sources for these applications.

The most challenging application that requires high power $(10 \mathrm{~W})$ with an extraordinary degree of purity for both the spatial and the temporal modes is gravitational-wave interferometry. ${ }^{1,2}$ The masteroscillator power-amplifier (MOPA) concept was proposed in 1995 by the Stanford group ${ }^{3}$ for the use in the American Laser Interferometer GravitationalWave Observatory (LIGO) because of its ability to scale the output power while maintaining the masteroscillator coherence and operational reliability. ${ }^{4}$ To reach the demanding spatial mode and temporal noise requirements these experiments require a combination of active stabilization and passive filtering of the laser source. $^{5}$ Fabry-Perot interferometers have been proposed as mode cleaners ${ }^{6,7}$ to filter the laser beam spatially and temporally before its introduction into the laser interferometer for gravitational-wave detection.

We report the use of a fixed-spacer triangular-ring cavity, called the premode cleaner, to filter the output of a $10-\mathrm{W}$ Nd:YAG MOPA $^{8}$ built by Lightwave Electronics Corporation under contract with the LIGO project. The master oscillator is a laser-diode-pumped monolithic Nd:YAG nonplanar ring oscillator (NPRO; Lightwave Electronics Model 126-1064-700). The master-oscillator output $(320 \mathrm{~mW})$ is transmitted through an electro-optic modulator, a pair of cylindrical lenses to circularize the beam, and isolation optics before the output enters the power amplifier. The power amplifier consists of four Nd:YAG laser rods, each pumped by two diode bars operating at a power of $16 \mathrm{~W}$ each. The double-passed amplifier has a power gain of 33 and generates $10 \mathrm{~W}$ of output power.

0146-9592/98/211704-03\$15.00/0
The 10-W laser beam is mode matched into the premode-cleaner cavity as shown in Fig. 1. The premode cleaner consists of three mirrors in a ring configuration with an opening angle of $87.13 \mathrm{deg}$ and a round-trip length of $42 \mathrm{~cm}$, similar to the design of Uehara et al. ${ }^{9}$ Two plane mirrors are attached (with epoxy) to a fused-silica prism, which is itself attached to a $17.5-\mathrm{cm}$-long rigid fused-silica spacer. Both the spacer and the prism have holes with sufficient clearance to avoid clipping the beam. A curved mirror with a 1-m radius of curvature is attached to a piezoelectric transducer, which is then attached to the other end of the fused-silica spacer. A finesse of 220 was measured for the $p$ polarization, whereas the finesse for the $s$ polarization was 4440 . For this experiment we used the $p$ polarization, for which we obtained $94 \%$ power throughput for the $\mathrm{TEM}_{00}$ Gaussian mode.

We used several techniques to compare the spatial profile of the 10-W laser beam before and after filtering. First a small fraction of the light was picked
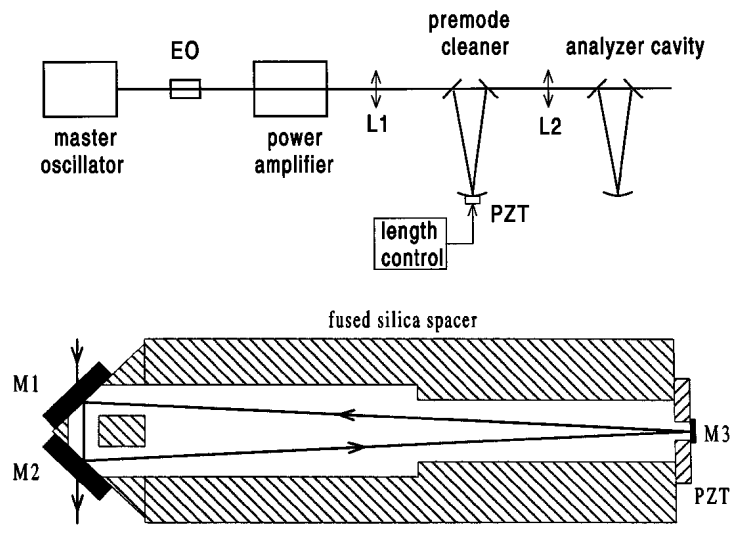

Fig. 1. Schematic of the experimental setup and the premode-cleaner Fabry-Perot ring cavity design. A detailed description of the length-control scheme is given in the text. EO, electro-optic modulator; PZT's, piezoelectric transducers; L1, L2, mode-matching lenses; M1, M2, 25.4-mm-diameter plane mirrors; M3, 7.75-mm-diameter mirror with 1-m radius of curvature. Cavity round-trip length, $42 \mathrm{~cm}$.

(C) 1998 Optical Society of America 
off before and after the premode cleaner and sent into a beam analyzer (BeamScan, Photon, Inc.). Figure 2 shows the measured beam profiles in the horizontal and vertical directions. The data of Fig. 2(a) were taken before the premode cleaner. The faint curves are calculated best-fit Gaussian profiles, the parameters of which were obtained with a nonlinear fit. The beam profiles have a good overall Gaussian character, but deviations between measurement and fit are evident. Similar distortions as well as the notable difference of the horizontal and vertical beam diameters were measured in the NPRO master-oscillator spatial profile, although most of the NPRO ellipticity was corrected by the pair of cylindrical lenses. Figure 2(b) shows the measured beam profiles of the $7.6-\mathrm{W}$ laser beam following the premode cleaner. The measurements and best-fit curves are indistinguishable within the resolution of this plot. The residual between measurement and fit is less than $1 \%$ for each individual point, and the ellipticity is reduced to less than $1 \%$ difference in horizontal and vertical beam diameter.

We determined the residual power in the higherorder spatial modes of the $10-\mathrm{W}$ laser by scanning the premode cleaner over one free spectral range and measuring the transmitted and reflected light. This procedure corresponds to measurement of the laser beam profile expanded into the normal modes of the cavity. Figure 3 shows a measurement of the relative power transmitted through the premode cleaner as a function of the premode cleaner's cavity length. The $\mathrm{TEM}_{00}$ mode is the dominant mode, and the distance between the two $\mathrm{TEM}_{00}$ peaks corresponds to one free spectral range of $713 \mathrm{MHz}$. Pictures taken with a CCD camera of the $\mathrm{TEM}_{00}$ mode and the largest non$\mathrm{TEM}_{00}$ mode are shown as insets. By scanning the premode cleaner length slowly over a free spectral range of the cavity we measured the transmitted power and the relative frequency position of the five most significant non-TEM ${ }_{00}$ modes with respect to the $\mathrm{TEM}_{00}$ mode. Adding all these contributions, we found $9.3 \%$ of the MOPA output power in higher-order modes. We obtained the same result by measuring the reflected power instead of the transmitted power while scanning the premode-cleaner length over a free spectral range.

The cavity must be kept in resonance with the laser when the cavity is used as a premode cleaner. We locked the cavity resonance frequency to the laser frequency, using the Pound-Drever-Hall technique. ${ }^{10}$ The light was phase modulated with the electro-optic modulator following the master oscillator where the light power was less than $350 \mathrm{~mW}$. At this lower power we were able to use a small-aperture modulator $(2 \mathrm{~mm})$ and still operate at low intensity in the crystal. We used a modulation frequency of $20 \mathrm{MHz}$ and a modulation index of 0.06 . The phase modulation sidebands as well as the carrier were amplified and then mode matched to the premode cleaner. The signal of the fast photodiode measuring the reflected light power was then demodulated and filtered to provide an error signal for the length-control servo system. The piezoelectric transducer was used as the actuator of this servo system to lock the cavity to the laser. The bandwidth of this loop was limited by a mechanical resonance of the fused-silica spacer at $9 \mathrm{kHz}$.

With this servo system in place we measured $7.6 \mathrm{~W}$ of transmitted light power for an incident power of $9.1 \mathrm{~W}$. After we account for the power in non-TEM ${ }_{00}$ modes and the phase modulation sidebands, this transmitted light power corresponds to $94 \%$ throughput of the light power in the $\mathrm{TEM}_{00}$ mode. To get an accurate measurement of the spatially filtered laser profile we mode matched the light transmitted by the premode cleaner into a second ring cavity. By scanning the length of the second cavity we determined that $0.1 \%$ of the light power after the premode cleaner was in non-TEM 00 modes. This measurement confirmed the calculated residual power in higher-order modes.

In addition to performing spatial mode filtering, the premode cleaner reduces the technical power noise of the 10-W laser, which is important for experiments that require a shot-noise-limited laser source. The gravitational-wave interferometers, for example, use heterodyne techniques that transform
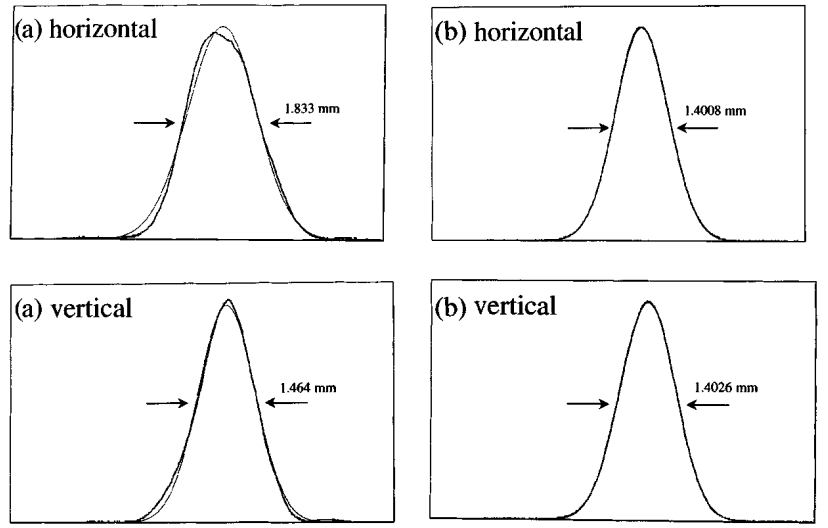

Fig. 2. Measured horizontal and vertical beam profile of the 10-W laser and best-fit Gaussian profiles: (a) before the premode cleaner and (b) following the premode cleaner, where measurement and fit agree to within the pen width.

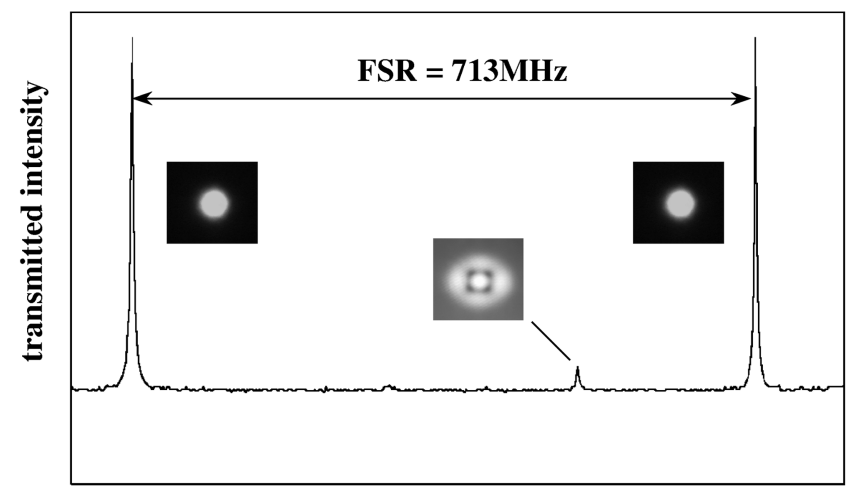

cavity length

Fig. 3. Transmitted power of the 10-W laser through the premode cleaner as a function of the cavity length. Insets, pictures (taken with CCD camera) of the dominant $\mathrm{TEM}_{00}$ mode and the largest non-TEM $\mathrm{T}_{00}$ mode. The higher-order mode content before the premode cleaner was $\approx 10 \%$, whereas following the premode cleaner the relative power in non- $\mathrm{TEM}_{00}$ modes was reduced to $10^{-3}$. FSR, free spectral range. 


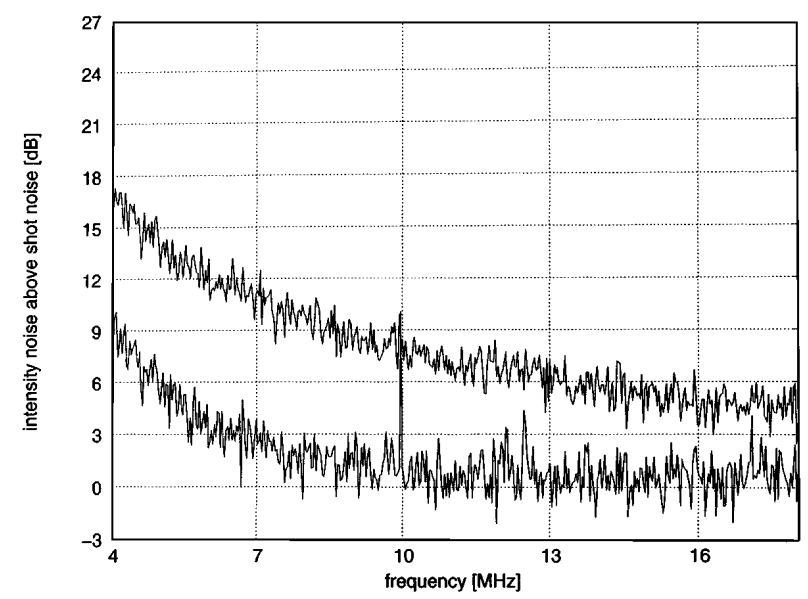

Fig. 4. Power noise of the $10-\mathrm{W}$ laser relative to the shot noise at $50 \mathrm{~mA}$ of detected photocurrent. Upper curve, measurement before the premode cleaner; lower curve, measurement following the premode cleaner. The power noise following the premode cleaner is shot-noise dominated above $7 \mathrm{MHz}$ and less than $1 \mathrm{~dB}$ above the shot-noise limit for frequencies higher than $10 \mathrm{MHz}$.

the gravitational-wave signal into the megahertz frequency band to achieve sensitivity limited by the shot noise of the detected light. Thus it is crucial to reduce the technical noise at the modulation frequency to the shot-noise limit. The LIGO gravitational-wave interferometer requires the power noise of the laser to be no more than $0.5 \%$ above the shot noise of $600 \mathrm{~mW}$ of detected light at $25 \mathrm{MHz}$. As an active feedback control loop cannot reduce the technical noise to this level, ${ }^{11}$ we used the premode cleaner to achieve this goal. The power noise filtering of a Fabry-Perot cavity can be described by $H_{T}(f)=1 /\left[1+\left(f / f_{c}\right)^{2}\right]^{2}$, where $H_{T}(f)$ is the power fluctuation transfer function for light transmitted through the cavity at Fourier frequency $f$ and where $f_{c}$ is the cavity half-width at half-maximum.

Figure 4 shows the relative power noise of the highpower MOPA laser relative to the shot-noise level for $50 \mathrm{~mA}$ of detected photocurrent before and after the premode cleaner. The power noise above shot noise is a measure of the fraction of technical noise that is present in the laser beam. Two noise sources are responsible for the technical noise in the MOPA before the premode cleaner (upper curve): the technical noise of the NPRO, which is amplified by the power amplifier, and noise introduced by the amplification process. ${ }^{12}$ The lower curve shows the filtered power noise following the premode cleaner. Using the calculated power noise transfer function of the premode cleaner, we expect $8.2 \mathrm{~dB}$ of power noise filtering at $4 \mathrm{MHz}$, which is in good agreement with the measurement. At $6 \mathrm{MHz}$ the power noise amplitude spectral density following the premode cleaner is $3 \mathrm{~dB}$ above shot noise, which indicates that the technical noise equals the shot noise of $50-\mathrm{mA}$ detected photocurrent. The peak at $10 \mathrm{MHz}$ is the filtered power fluctuation impressed by the electro-optic modulator as the result of a spurious $10-\mathrm{MHz}$ signal present in the rf-oscillator signal. This modulator was turned off while we measured the amplitude noise before the premode cleaner.
In conclusion, a 10-W laser-diode-pumped Nd:YAG MOPA was spatially and temporally filtered with a fixed-spacer Fabry-Perot ring cavity. This premode cleaner provides a light source with an excellent spatial profile and low power noise at radio frequencies. Both the MOPA design and the passive filtering offer the possibility to increase the laser power to $100 \mathrm{~W}$ and beyond. Although this light source was developed to meet the needs of high-sensitivity interferometry for the detection of gravitational waves, the demonstrated concept is versatile, and the premode cleaner can easily be implemented in different types of laser systems to yield spatial and temporal filtering.

We thank David Shoemaker and Stan Whitcomb for proposing the use of a premode cleaner as a passive power noise filter and for suggesting this collaborative research. B. Willke thanks the German Alexander von Humboldt-Stiftung for his Feodor-Lynen fellowship. This research is supported in part by the National Science Foundation (NSF) under cooperative agreement PHY-9210038 and NSF grant PHY-9630172.

\section{References}

1. A. Rüdiger, Nucl. Phys. B 48, 96 (1996).

2. A. Abramovici, W. E. Althouse, R. W. P. Drever, Y. Gürsel, S. Kawamura, F. J. Raab, D. Shoemaker, L. Sievers, R. E. Spero, K. S. Thorne, R. E. Vogt, R. Weiss, S. E. Whitcomb, and M. E. Zucker, Science 256, 325 (1992).

3. The MOPA concept for the LIGO gravitational-wave detector was proposed in the GALILEO proposal, NSFPHY9630172.

4. R. J. Shine, Jr., "A high-power, diode-laser-pumped solid-state laser for precision interferometry," Ph.D. dissertation (Stanford University, Stanford, Calif., 1995).

5. J. Hough, H. Ward, G. A. Kerr, N. L. Mackenzie, B. J. Meers, G. P. Newton, D. I. Robertson, N. A. Robertson, and R. Schilling, in The Detection of Gravitational Waves, D. G. Blair, ed. (Cambridge U. Press, Cambridge, 1991).

6. A. Rüdiger, R. Schilling, L. Schnupp, W. Winkler, H. Billing, and K. Maischberger, Opt. Acta 28, 641 (1981).

7. K. D. Skeldon, K. A. Strain, A. I. Grant, and J. Hough, Rev. Sci. Instrum. 67, 2443 (1996).

8. W. Wiechmann, T. J. Kane, D. Haserot, F. Adams, G. Truong, and J. Kmetec, in Conference on Lasers and Electro-Optics, Vol. 6 of 1998 OSA Technical Digest Series (Optical Society of America, Washington, D.C., 1998), p. 432.

9. N. Uehara, E. K. Gustafson, M. M. Fejer, and R. L. Byer, Proc. SPIE 2989, 57 (1997).

10. R. W. P. Drever, J. L. Hall, F. V. Kowalski, J. Hough, G. M. Ford, A. J. Munley, and H. Ward, Appl. Phys. B 31, 97 (1983).

11. C. C. Harb, M. B. Gray, H.-A. Bachor, R. Schilling, P. Rottengatter, I. Freitag, and H. Welling, IEEE J. Quantum Electron. 30, 2907 (1994).

12. W. M. Tulloch, T. S. Rutherford, E. H. Huntington, R. Ewart, C. C. Harb, B. Willke, E. K. Gustafson, M. M. Fejer, R. L. Byer, S. Rowan, and J. Hough, "Measurements of quantum noise in a cw laser-diodepumped Nd:YAG linear optical amplifier," submitted to Opt. Lett. 